\title{
La Evaluación Ambiental Estratégica (EAE) en Colombia: su adopción, criterios para la estructuración de su procedimiento administrativo y su aplicación a los planes de ordenamiento territorial
}

Jorge Gómez

\section{RESUMEN}

El propósito de esta investigación fue discutir algunos modelos formales del procedimiento político-administrativo para la Evaluación Ambiental Estratégica (EAE) en Colombia - la cual se aplica a nivel de políticas, planes y programas (PPP). A partir de los principios teóricos de la evaluación ambiental y de la experiencia en el país con la Evaluación de Impacto Ambiental - EIA(complementada con la corta historia de la aplicación de la EAE), se derivaron criterios para estructurar los pasos del procedimiento administrativo de este instrumento. Así mismo, se consideraron dos modelos para el procedimiento de la EAE a saber: uno similar al de la EIA clásica que se basa en el orden de la sucesión de pasos del procedimiento de la EIA aplicada en Colombia (para el caso de la EAE implica, en principio, poseer un borrador del diseño de las PPP para hacer la evaluación ambiental y, según los resultados, proponer las medidas y recomendaciones ambientales necesarias para modificarlas), y otro basado en la integración de la EAE acompañando desde su inicio el proceso de planificación de las PPP e introduciéndoles en este proceso de su diseño simultáneamente las modificaciones pertinentes desde la perspectiva ambiental. En el campo del ordenamiento territorial en Colombia, según el procedimiento existente y los determinantes para la planificación y concertación de los Planes de Ordenamiento Territorial (POT) del nivel local, el modelo más adecuado a aplicar es el segundo referido, es decir, el de la EAE integrada, en el cual se da la articulación simultánea entre las fases de la EAE con las etapas de planificación (diseño) del proyecto del POT.

\section{Palabras claves:}

Evaluación Ambiental Estratégica (EAE), Evaluación de Impacto Ambiental (EIA), políticas públicas, planes y programas, Procedimiento Político-Administrativo (PPA), ordenamiento territorial

“The Strategic Environmental Assessment in Colombia: Its Adoption, Criteria for the Structuring of its Administrative Procedure and its Application to the Spatial Plans"

* Doctor en Ingeniería (Área Planificación Ambiental). Coordinador y profesor de la Especialización enEducación y Gestión Ambiental de la Universidad Distrital Francisco José de Caldas. Director Grupo de Investigación Educación y Gestión Ambiental, neotropico@hotmail.com 


\section{ABSTRACT}

The purpose of this research was to propose formal models of political administrative procedure for the strategic environmental assessment (SEA) in Colombia, which applies at the level of policies, plans and programs (PPP). From the debate on the theoretical principles of the environmental assessment and from the Colombian experience with the Environmental Impact Assessment -EIA (complemented with the short history of the SEA), it is possible to obtain from there criteria to sructurise the different steps of the administrative arrangement and process of the SEA. From these principles it is possible to derivate various models for the SEA, considering basically two of them: One that is similar to the classical EIA, that presumes to follow in a similar fashion the order of succession of steps of the procedure of the EIA as applied in Colombia (that implies at least having a draft of the design of the PPP, in order to make it go through the environmental assessment and from its results obtain the measures and recommendations that are to be considered) and, another one based on the integration of the SEA accompanied by the planning and design process of the PPP and simultaneously introducing in them the pertinent changes from the environmental perspective. In the particular field of spatial planning in Colombia and according to existing procedure or arrangement and its determinant key points for the planning and formal agreement of the Territorial Organisation Plans the most adequate model to be applied would be the second referenced one, in other words, the integrated SEA, in which there is the simultaneous articulation among the phases of the SEA with the steps of the planning (design) of the Territorial Organisation Plan.

\section{Keywords}

Strategic Environmental Assessment, Environmental Impact Assessment, public policies, policies, plans, programms, Environmental Administrative Procedures, spatial planning.

Abreviaturas: PPP: Política(s) y/o Plan(es) y/o Programa(s); EAE: Evaluación Ambiental Estratégica; EIA: Evaluación de Impacto Ambiental; AA:
Autoridad Ambiental; AAC: Autoridad Ambiental Competente; AC: Autoridad Competente; POT: Plan de Ordenamiento Territorial.

\section{Introducción}

El propósito central de este proyecto de investigación fue analizar las posibilidades para la institucionalización de la Evaluación Ambiental Estratégica (EAE) en Colombia, cuyo ámbito de aplicación es el de las PPP (etapas de toma de decisión estratégicas anteriores a la de proyectos). Estos últimos concretizan las PPP y representan -teóricamentela última unidad en el proceso de planificación. Si bien a este nivel también se aplica la evaluación ambiental -denominada comúnmente Evaluación de Impacto Ambiental (EIA)-, el margen de toma de decisiones a esta escala no es tan amplio; las decisiones tomadas en los niveles superiores de este proceso ( $\mathrm{PPP}$ ) condicionarán las actuaciones de los niveles posteriores. La EAE implica, además de un incremento en el trabajo y los costos para las instituciones, mayor presión para el sistema de planificación que lo soporta. No obstante, supone varias ventajas ${ }^{1}$ :

- Asegura que los responsables de las decisiones a nivel de PPP incluyan más sistemáticamente los aspectos ambientales en los procesos de planificación y de toma de decisiones.

- Apoya el trabajo de la(s) Autoridad(es) Competente(s) (AC) para que haya un trato semejante con todos los promotores de proyectos en un territorio; facilita que en el marco de una EAE, puedan fijarse -de forma más estandarizada- valores límites e indicadores para la EIA (nivel de proyectos).

- Permite el trabajo en las escalas posteriores del proceso de planificación ya que aporta datos para las etapas que se desarrollan des-

1 Handbuch Strategische Umweltprüfung (HB SUP) (Manual de la EAE) Wien: Institut für Techikfolgen - Abshätzung, 1997/2000/2001/2003/2004/2006, pag. 1-3, 1-4 
pués (como la de proyectos). En ese sentido puede contribuir a la disminución de demoras en los procedimientos administrativos de EIA debido a las directrices y los datos que aporta -lo que a su vez puede repercutir en la disminución de costos de la realización de estos estudios.

- Al facilitar el trabajo para la etapa posterior de la EIA (nivel de proyectos), puede hacer más atractivo un territorio para la inversión.

- Estimula la participación de los diferentes actores, eleva la transparencia y responsabilidad en el proceso de toma de decisiones, facilita el manejo del conflicto y mejora la aceptación de las decisiones a nivel de PPP.

Para la institucionalización de este instrumento se requiere estructurar un procedimiento político-administrativo que establezca las reglas de juego entre los actores involucrados y permita, a su vez, el manejo del conflicto entre ellos. Lo anterior implica profundizar en criterios como: su articulación con los procedimientos de planificación existentes para las PPP; la determinación de su ámbito deaplicación en las PPP; el alcance de la evaluación (definición de aspectos a ser evaludos, metodologías, selección de alternativas a considerar, etc.); instituciones responsables del manejo del instrumento; participación del público e intervención de otras instituciones en este proceso; revisión e integración de los resultados de la evaluación en el diseño de PPP, entre otras. El modelo de procedimiento de EAE que podría adoptarse está sujeto a factores como el contexto en que surgen las PPP, así como a sus modalidades, características, estructuración y componentes del proceso de formulación, ejecución y control (seguimiento, evaluación), y del diagnóstico, objetivos, estrategias, propuestas, etc. de los PPP. También depende de las condiciones específicas para la gestión ambiental; es decir, del marco político-administrativo, económico, social, ecológico y cultural existente en Colombia, que sirve de base, a su vez, para la EAE. Para la EAE se puede tener como referencia EIA, instrumento sobre el cual ya se tiene una larga experiencia en el país y puede servir (con sus respectivas modificaciones) como uno de fundamento para la discusión de la estructuración del procedimiento administrativo de la EAE en Colombia. Lo anterior teniendo en cuenta que el sistema de EIA vigente (con sus virtudes y problemas) ha sido fruto de la decantación de varios años y es el reflejo final de las condiciones geográfico-naturales; de la tradición y cultura jurídica; estructura y cultura institucional, condiciones políticas, etc., aspectos que también influirán en la estructuración de la EAE.

En la discusión sobre la EAE, además de los planteamientos que hacen referencia al "qué" (qué tipos de PPP se pueden incluir y qué modalidades generales de EAE se pueden aplicar), es importante responder al "cómo", o sea, a la forma concreta o modelo específico de implementar este instrumento considerando las especificidades de un ámbito de PPP determinado. Para estos efectos, en este trabajo se examinan los tipos de modelos de procedimiento administrativo con el fin determinar el más adecuado para aplicar -considerando un área de PPP específica: la del ordenamiento territorial.

Con base en lo anterior, y para analizar las posibilidades de estructuración del procedimiento político-administrativo de la EAE en Colombia, se elaboró un estudio acerca del contexto y las características generales de PPP en el país considerando aspectos centrales como el desarrollo del ciclo político y las características y relaciones entre las PPP y los contextos institucionales en que se desarrollan. También, se profundizó en los elementos principales del procedimiento político administrativo de la EAE; partiendo de principios generales que sirven como referentes a la evaluación ambiental en general -considerando las particularidades de la evaluación aplicada a PPP-, se derivaron criterios que pueden ser tenidos en cuenta como primera aproximación para orientar la estructuración de su procedimiento. De acuerdo con esta reflexión se plantean dos modelos de procedimientos administrativos generales de $\mathrm{EAE}$ que podrían considerarse para ser instaurados en 
Colombia y, así mismo, se verifica su aplicabilidad general en el área del Ordenamiento Territorial -específicamente en los Planes de Ordenamiento Territorial (POT) en el nivel local, área seleccionada por su condición estratégica para el desarrollo del país.

Este proyecto corresponde básicamente a un tipo de investigación empírica-analítica y descriptivaexplicativa. Se recurrió, como sustento informativo, tanto a la recopilación bibliográfica como a una entrevista informal con un actor institucional determinante en la introducción de la EAE a Colom$\mathrm{bia}^{2}$. El proceso de investigación se realizó continuamente durante sus diferentes fases (recolección de información, organización e interpretación) de acuerdo a categorías conceptuales propuestas en el marco teórico y en el planteamiento del problema. Así mismo, fueron desarrollados diversos procedimientos de análisis e interpretación con el objetivo de confrontar permanentemente los referentes conceptuales para la EAE y EIA, y el conocimiento y la experiencia del autor sobre la temática de esta propuesta de investigación.

\section{Caracterización de las políticas públicas en Colombia y su relación con la EAE}

En Colombia ha habido una paulatina institucionalización de las políticas públicas. Cada vez se ha intentado formalizar y racionalizar más las competencias y las funciones de las instancias responsables de liderarlas, así como de definir mejor sus instrumentos de planificación, avanzar en su desarrollo práctico y mejorar las instancias de participación de los diferentes agentes involucrados, etc. Sin embargo, no siempre se ha dado una formulación explícita de la política pública que sigue cada organismo del sector público; frecuentemente la misma normatividad que la crea, en los apartes referentes a su finalidad y funcionamiento, sirve como referente explícito de la política a desarrollar por parte de cada sector. Naturalmen-

2 Ministerio de Ambiente, Vivienda y Desarrollo Territorial (MAVDT) te, cada organismo tiende al cumplimiento de sus funciones adelantando una serie de programas y proyectos que, implícitamente, son un referente de la política que están ejecutando en la praxis ${ }^{3}$ Todavía, en el sector público, se presenta una proliferación de políticas que formulan y ejecutan una variedad de organismos -muchas veces sin suficiente coordinación- lo cual afecta su eficacia $y$ eficiencia ${ }^{4}$.

Algunas características centrales de las políticas públicas en Colombia, relevantes para la estructuración de la EAE, son:

- Es habitual que no haya una diferenciación muy formal entre políticas, planes y programas. Si bien teóricamente se plantea que en las decisiones estratégicas el nivel de políticos ${ }^{5}$ es el más general, esta organización jerárquica se desdibuja frecuentemente en la práctica. Dicha situación puede obedecer a factores como las características y las relaciones entre PPP y la diversidad de contextos institucionales, o a los niveles territoriales en los que se desarrollan. Por otra parte, las características del entorno donde se gestan las políticas dificultan la aplicación de una evaluación ambiental (instrumento que generalmente ha sido diseñado para procesos de toma de decisiones en los que predomina una racionalidad técnica). Por eso su aplicación por lo regular es más favorable en lo que concierne a planes y programas; por ser niveles un poco más instrumentales, permiten en principio, que prime mucho más ( que el nivel de políticas), la racionalidad técnica mencionada.

3 Apoyándose en CANO MOTTA, Augusto "La Planeación en Colombia" Mimeo Bogotá: Departamento Nacional de Planeación DNP, 1967 citado en MORCILLO, Pedro Pablo "La Planeación en Colombia- Historia, Derecho y Gestión” Bogotá: Universidad Piloto, 2002, pag. 263

4 Apoyándose en MORCILLO, Pedro Pablo, Op. Cit., pag. 324

5 Los lineamientos de una política teóricamente se deben concretar a nivel de un plan, el cual a su vez debería contener diferentes programas que se operacionalizan por medio de diferentes proyectos 
- La falta de acuerdos, sumado a los vacíos normativos y a la ausencia tanto de una estandarización sistemática de los contenidos como de relaciones entre las áreas y los sectores e instrumentos de planificación de política pública, conduce a que éstas presenten una gran diversidad. Si bien existe una coordinación y homogenización mayor en ciertos campos (planeación nacional de desarrollo, planes de desarrollo territoriales y planes de ordenamiento territorial del nivel local), otros tantos presentan gran heterogeneidad en cuanto a sus dimensiones espaciales, instrumentales, presupuestales, horizontes de tiempo y mecanismos de coordinación. Dicha diversidad y confusión condicionan los procedimientos y las metodologías que se pueden desarrollar en el marco de una EAE, pues es difícil implementar una reglamentación detallada que corresponda a una situación de políticas públicas relativamente estandarizadas en su morfología.

- La fragmentación institucional, que se presenta con frecuencia en todos los niveles del aparato político-administrativo, se evidencia en las iniciativas e instrumentos empleados por cada uno de ellos (las PPP, por ejemplo). Así mismo, puede considerarse la existencia de múltiples situaciones en cada esfera territorial y área del desarrollo; la capacidad política, técnica y presupuestal es diferente en en los niveles al interior de la escala territorial. Incluso, en las capacidades de cada uno de ellos predominan diferencias marcadas. La heterogeneidad anterior se constituye en un reto en el momento de introducir la EAE ya que su adopción debe ajustarse a esta realidad institucional -desigual y compleja- y superar las dificultades que representa la fragmentación institucional en todo sentido. Entre tanto, su compartimentalización, debido a los celos competenciales y al enfoque sectorial de muchas de éstas instituciones, genera resistencias para introducir la EAE con la cual se buscaba en principio transversalizar las consideraciones ambientales.

\section{Síntesis de los criterios a considerar para la estructuración del procedimiento general de la EAE}

A partir de la discusión del contexto y las características de las PPP, así como de los principios generales para la estructuración del procedimiento administrativo de las evaluaciones ambientales y la experiencia específica con la EIA (y de las experiencias pilotos con la $\mathrm{EAE}$ ); es posible enumerar los siguientes criterios generales como referentes para la EAE en Colombia:

- Relación con los procedimientos de planificación existentes: la EAE, para que cumpla a cabalidad con el principio de prevención, debe efectuarse en una fase previa a la ejecución de las PPP, es decir, en el momento de la formulación de éstas con fin de que los resultados de la evaluación puedan ser incluidos en el diseño de las PPP.

- Campo de aplicación: la introducción de un listado taxativo de PPP sería lo más conveniente; es un enfoque que, pese a su inflexibilidad, permite estructurar el campo de aplicación de la EAE con claridad. Para las actuaciones no incluidas en el listado se podría analizar (caso por caso y de acuerdo a criterios específicos) si la PPP requiere evaluación. En cuanto a la adopción de la EAE debería considerarse -teniendo en cuenta la poca experiencia con relación a este tipo de evaluaciones - un grupo pequeño de PPP y así evitar una sobrecarga en las autoridades responsables de su procedimiento administrativo. Además, podrían seleccionarse áreas de políticas públicas en las que se presente un escalonamiento claro de PPP y/o zonas estratégicas por su impacto en el territorio y las potencialidades de aprendizaje para a los diferentes actores.

- Responsable del manejo del procedimiento administrativo: en el caso colombiano, la responsabilidad del manejo del instrumento recae en las AAC; básicamente el Ministerio de Ambiente, Vivienda y Desarrollo

REVISTA CIENTÍFICA / ENERO -DICIEMBRE DE 2010 / No. 12 / BOGOTÁ, D.C. 
Territorial (MAVDT) para las PPP del nivel nacional y las Corporaciones Autónomas Regionales (CAR) para las PPP departamentales o locales. Dichas instituciones, por su misión fundamental en el área ambiental, deben representar a cabalidad los intereses ambientales $y$, en este caso, responsabilizarse para otorgar un manejo adecuado al proceso de la EAE (especialmente para la decisión final).

\section{- Coordinación con otras instituciones:} debería considerarse una amplia intervención de instituciones a lo largo del proceso -siempre y cuando se amerite- para hacerlas partícipes en momentos esenciales. Lo anterior se lograría considerando una fase inicial en la cual se involucren cada una de estas instituciones. De esta manera, pueden escucharse y considerarse, de forma oportuna, diferentes comentarios en torno a la EAE que se va desarrollar. Posteriormente, cuando se haya efectuado el EAE y se cuente con el reporte de los resultados, se contemplaría nuevamente la intervención de las instituciones.

- Alcance: esta fase temprana de participación del público e intervención de otras instituciones, permite plantear inquietudes y aportar insumos para definir los aspectos a considerar y las metodologías a seguir en la evaluación ambiental. La participación, para los agentes institucionales claves, es indispensable; mientras que para el público en general y otros actores institucionales serían (participarían sólo por petición).

- Participación del público: en lo posible se debería buscar una amplia participación del público, tanto afectados como interesados por la PPP. Además, como regla general, debe considerarse que cuanto más temprano se inicien los procesos de participación, mayor facilidad habrá para modificar un diseño o incorporar -en la discusión - aspectos que el público considere significativos o potencialmente conflictivos ${ }^{6}$. Por ende (tal como se planteó en el ítem anterior), es importante considerar la fase de alcance de la EAE para examinar las inquietudes del público en el proceso de evaluación.

Por otra parte, para desarrollar procesos de participación efectivos, se requiere como condición fundamental que el público esté bien informado. Esto implica la garantía del acceso a información relevante para fines evaluativos (incluyendo documentos, reportes y estudios generados en este proceso). Así mismo, para que el intercambio sea fluido, será fundamental un proceso comunicativo idóneo con el público; lo anterior implica el uso adecuado de los medios de comunicación según las características culturales, sociales y geográficas del público. Entre tanto, deben tenerse en cuenta algunas reglas para que la institución responsable se ocupe de los comentarios y las observaciones recogidas. A su vez, los motivos por los cuales se toman las decisiones en el marco de la EAE (teniendo como parte los insumos para estas decisiones los comentarios aportados por el público), deben ser fundamentados $\mathrm{y}$ dados a conocer.

En Colombia, los mecanismos de participación del público en procedimientos administrativos ${ }^{7}$ cumplen con gran parte de los criterios mencionados. Su aplicación en el marco de las EAE, dependería, en principio, de la introducción jurídica del procedimiento administrativo -aunque también lo complejizarían. Se requeriría, además, de una cierta estandarización de los procesos de toma de decisiones de las PPP sujetos a EAE que garantizara momentos precisos y apropiados para aplicar parte de estos instrumentos de participación (considerando especialmente las exigencias para desarrollar una audiencia

6 Ver a este respecto a OÑATE, Juan y Otros, Op, Cit., pag. 127

7 Intervención en Procedimientos Administrativos, $\mathrm{Au}$ diencia Pública Ambiental, Consulta a Comunidades Indígenas y Negras. Otro mecanismo que se puede aplicar en este contexto: Derecho de Petición de Información 
GRÁFICA 1. PROCEDIMIENTO GENERAL PARA LA EAE (Modelo 1 basado en la EIA clásica - tipo colombiano)

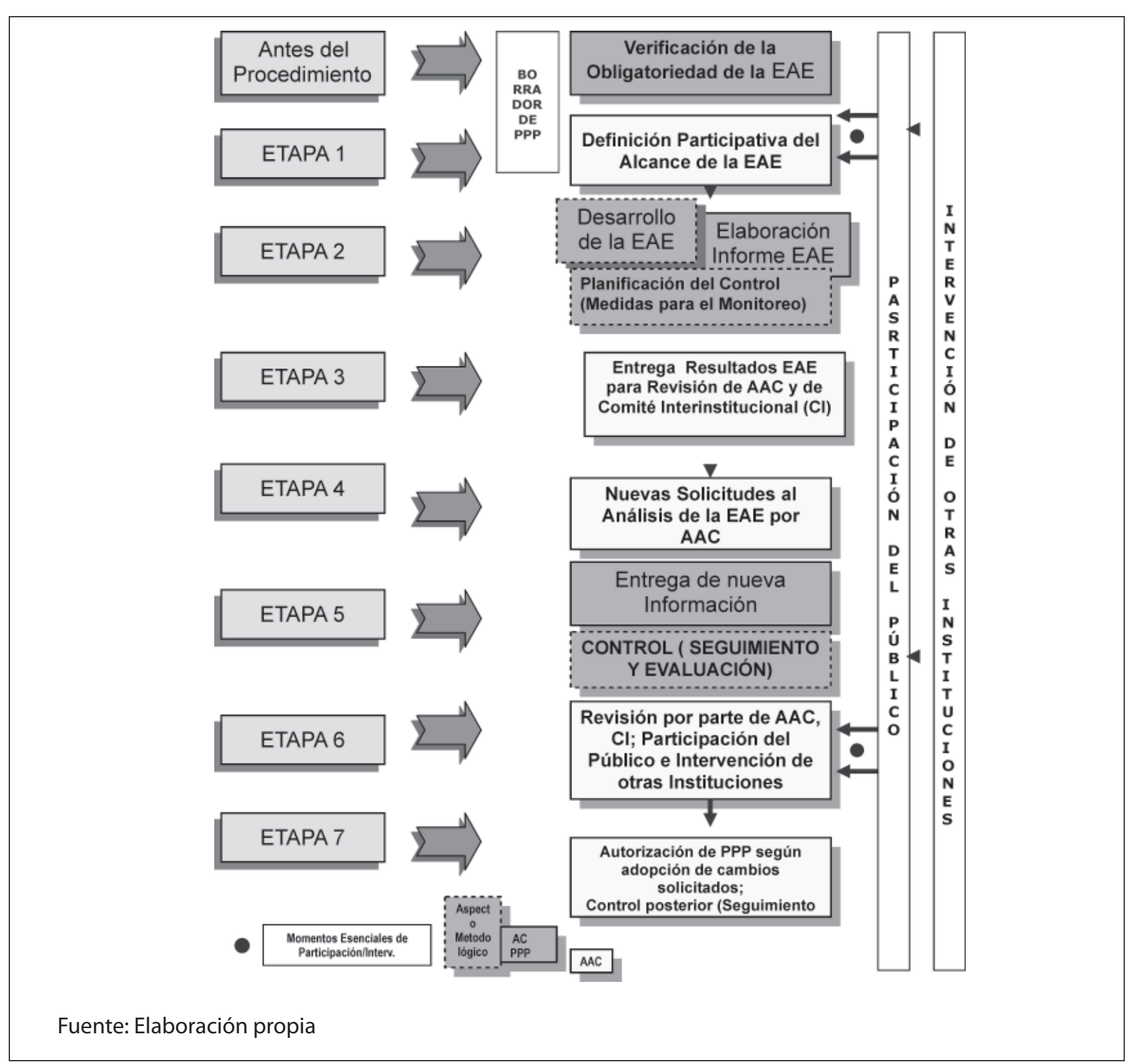

ambiental y la consulta previa a comunidades indígenas y negras). Sin embargo, los mecanismos analizados no constituyen los únicos arreglos de participación posibles, ya que pueden trabajarse otras de formas (talleres, reuniones, etc.). Si existen procedimientos legalmente establecidos, o espacios no formales de participación en el proceso de formulación de las PPP, se haría posible la racionalización del proceso integrando la participación para la EAE con estos espacios existentes.
- Revisión e integración de los resultados de la evaluación en la toma de decisiones: la responsabilidad de las decisiones sobre la aceptación o el rechazo de las medidas, y por tanto las PPP, debería de recaer -teóricamente- en un ámbito político y no tan técnico; especialmente si las PPP son de nivel jerárquico elevado. No obstante, dada la baja representación de los intereses ambientales con relación a los económicos y sociales, sería conveniente que la $\mathrm{AAC}$ tuviera la potes- 


\section{GRÁFICA 2. PROCEDIMIENTO GENERAL PARA LA EAE (Modelo 2 integrado al Proceso de Planificación de PPP)}

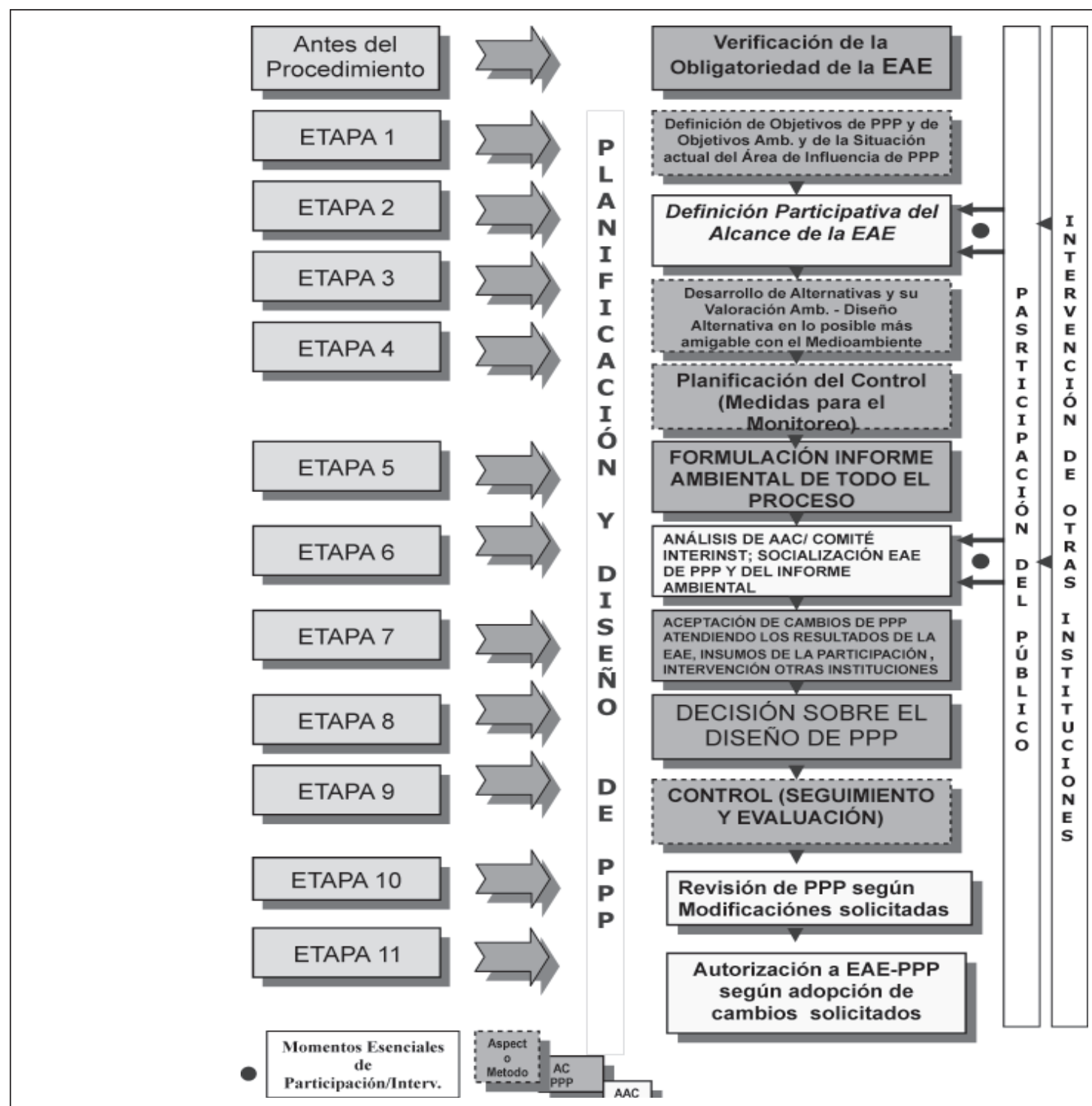

Fuente: Elaboración propia

tad de aprobar o no las PPP desde la óptica de la EAE. Esto garantizaría, en principio, una mejor integración de las recomendaciones ambientales de la EAE en las PPP.

En este trabajo se proponen dos modelos básicos de procedimientos generales de EAE: el primero es similar al modelo clásico de la EIA y cumple las etapas del prototipo colombiano (cabe anotar que se modifican algunas partes y se adicionan pasos) (gráfica 1); y el segundo, por su parte, se encuentra basado en la integración del proceso de la EAE con el del diseño de las PPP (gráfica 2). En ambas gráficas se representan esquemas básicos de las diferentes etapas del procedimiento de la EAE -algunos pueden sucederse de forma simultánea o algunas pequeñas con variaciones. El modelo 1 presupone la existencia de un borrador de PPP sobre el cual se debe realizar la evaluación; mientras que en el modelo 2 la EAE se desarrolla simultáneamente con la planificación y el diseño de las PPP. Los dos procedimientos incluyen la verificación previa del 
proceso de la EAE para la PPP de acuerdo a listados taxativos y/o criterios. Entre tanto, aunque se concibe la participación amplia del público, por razones logísticas y de capacidad institucional debería integrarse en momentos precisos y en ciertas etapas del procedimiento. En ambos modelos se señalan las exigencias mínimas de participación del público y de intervención de actores institucionales claves y de otras instituciones interesadas. Así mismo, se considera esencial la inclusión de organismos determinantes, la participación (optativa) del público y la cooperación (optativa) de otras instituciones tanto en fases tempranas como posteriores al proceso.

Por su parte, mientras que la participación e intervención en fases posteriores de los procedimientos de los instrumentos de planificación (PPP, evaluaciones ambientales, etc.) son habituales en Colombia, la participacón e intervención en fases tempranas crea, por lo general, resistencias en las $\mathrm{AC}$ responsables de manejar el proceso debido a complicaciones logísticas, costos, y dilaciones de tiempo, entre otras. No obstante, su buena aplicación puede contribuir a la legitimación del proceso de toma de decisiones.

En ambos modelos, la responsabilidad sobre la mayoria de las etapas del procedimiento de las EAE le correspondería a la AAC y a la AC de la PPP la realización de pocesos de la EAE más de tipo metodológico. Así mismo, en los dos ejemplos, la decisión final la toma la AAC y no la AC de la PPP.

\section{Análisis sobre la aplicabilidad de los modelos de procedimientos de EAE propuestos}

Los modelos presentados corresponden a esquemas genéricos que sirven como orientación para introducir el procedimiento administrativo de la EAE. A pesar de las diferencias, presentan similitudes en la denominación y en los propósitos de las etapas. No obstante, el modelo 1 -basado en la EIA clásica- supone una demanda de tiempo mayor; no se desarrolla sincrónicamente con toda la fase de formulación de PPP, sino, básicamente se debe tener la propuesta ( o borrador) de PPP para hacerle la evaluación. El modelo 2 acompaña la totalidad del proceso de planificación y diseño de PPP y permite tanto una integración más estrecha de la EAE como un desarrollo simultáneo de los pasos de PPP con los de la evaluación.

La aplicación de uno u otro modelo de EAE está sujeta a que se cumplan las condiciones apropiadas en un campo determinado de PPP que favoreciera su adopción. En principio, sería más fácil articularlos en un ámbito de PPP cuyos fundamentos legales fuesen generales y que no estructuraran un procedimiento específico mediante pasos y tiempos rígidos para la formulación de la PPP -en muchos casos no existe una estructura legalmente formalizada las PPP lo cual facilitaría laintegración de la EAE. Sin embargo, así no se tenga este procedimiento, se requiere de todas maneras partir de la dinámica del proceso de formulación de PPP para hacer la adaptación respectiva del procedimiento de la EAE.

Con relación, la inclusión de procesos de participación amplias que implica articular más etapas o pasos a estos modelos lo cual conlleva a costos y tiempos mayores -en comparación con otros procedimientos con menos etapas y con alcance más limitado de la participación del público y la intervención de otras instituciones-; esta podría contribuir para el mejoramiento de las condiciones de concertación, la facilitación del manejo de los conflictos y el posicionamiento de la transparencia en los procesos de formulación de PPP con relación a los aspectos ambientales. Estos niveles de decisión (más elevados que el de los proyectos) no siempre cuentan con espacios suficientes de participación para los diferentes actores y se da también la discrecionalidad en las decisiones por parte de las instituciones responsables del manejo de PPP. Con este procedimiento, al menos desde el punto de vista ambiental, las decisiones estarían -en principio- más permeadas por las opiniones y los comentarios aportados en los procesos de participación y de intervención. 


\section{GRÁFICA 3. FASES DE FORMULACIÓN Y EJECUCIÓN DEL POT ( LEY 388 DE 1997)}

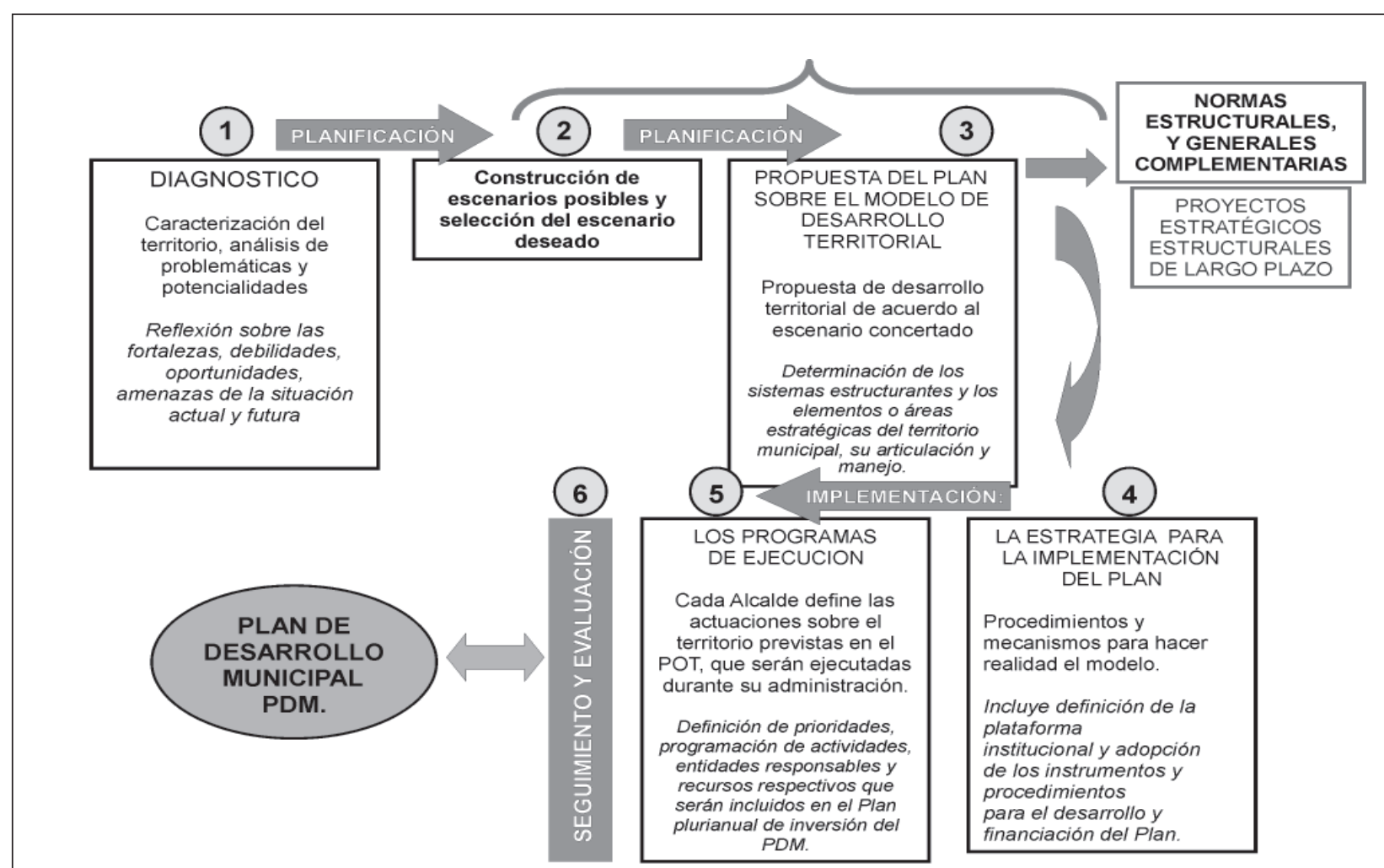

Fuente: MINDESARROLLO con modificaciones, 2001

La pregunta que surge de acuerdo a los propósitos de este trabajo es: ¿cómo articular el modelo de la EAE a los procedimientos de los POT, garantizan de la inclusión e influencia de los resultados de la evaluación en la concepción y diseño de estos planes? Además cumpliendo en lo posible con los criterios ya discutidos para este proceso. Para analizar las posibilidades de aplicación de los modelos de EAE propuestos en el ámbito específico de los POT, se debe examinar la situación particular del campo de ordenamiento territorial, revisar los modelos de EAE propuestos y examinar cuál de éstos es el más adecuado para integrarlo. La propuesta que se hace a continuación parte de los elementos formales que se infieren a partir de la reglamentación del ordenamiento territorial (Ley 388 de 1997) con relación a las etapas usuales para la formulación y concertación de los POT (gráficas 3 y 4); proponiéndose un esbozo de la forma de articulación de la EAE a este proceso (gráfica 5). Como se observa de las gráficas 3 y 4, existe una reglamentación relativamente detallada acerca del procedimiento para la formulación de los POT (a este proceso formalizado debe adaptarse el procedimiento de la EAE).

La articulación del procedimiento de la EAE al de los POT, debe considerar, como punto central, la posibilidad de incluir los resultados de la EAE en el plan, es decir, éstos deberían estar disponibles en el momento en que todavía puedan integrarse y realizarse cambios pertinentes en estos planes. De acuerdo al actual procedimiento de los POT, en la fase de concertación con la Autoridad Ambiental (AA) deberían estar incluidas estas recomendaciones ( de la EAE) para así constituirse en factores estructurantes y en elementos para la discusión del plan en el período de participación e intervención de otros actores y de concertación con otras insti- 


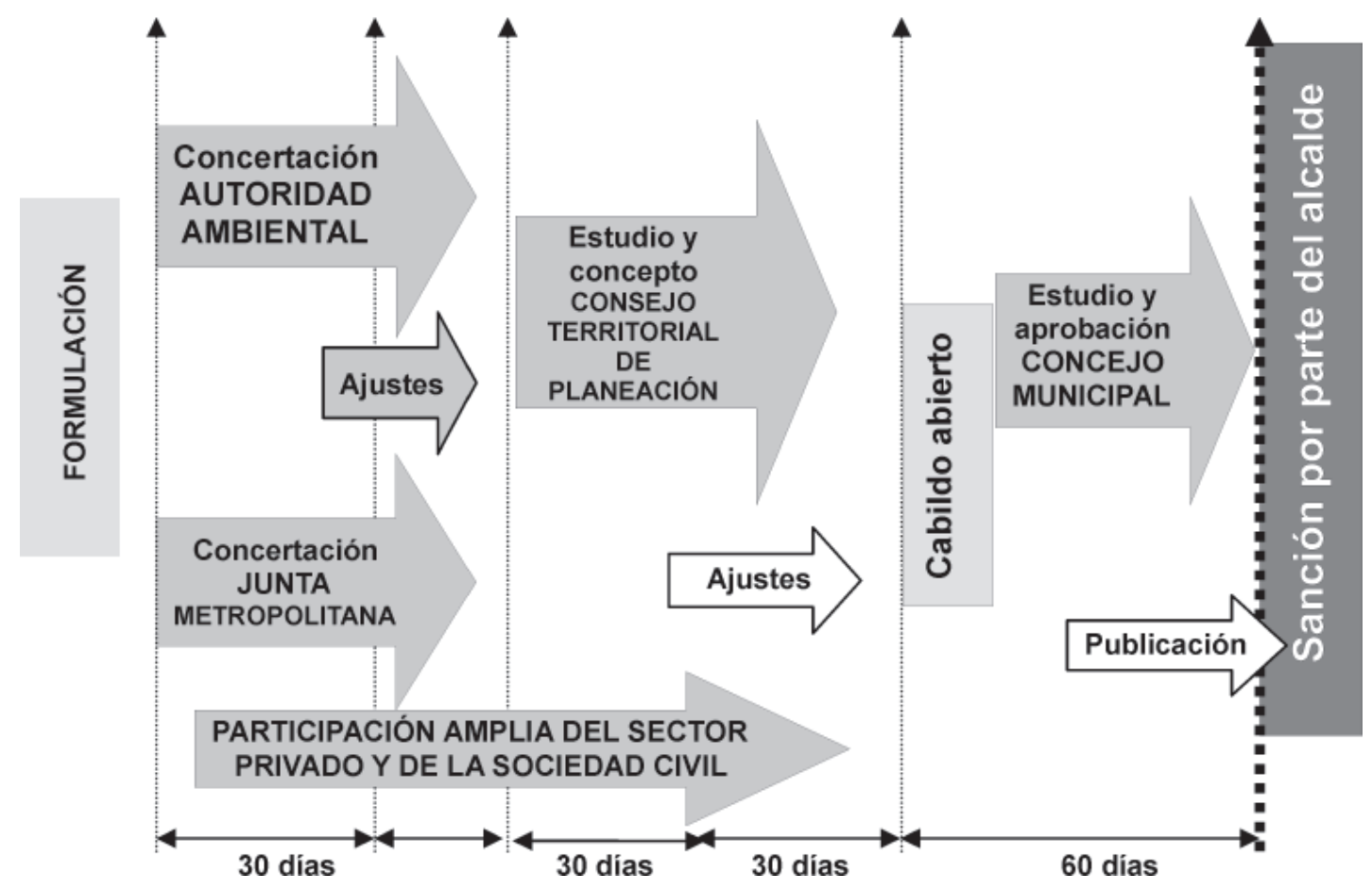

Fuente: MINDESARROLLO con modificaciones, 2001

tuciones, que se desarrolla simultánea o posteriormente al momento en que la AA realice la revisión del $\mathrm{POT}^{8}$. Para dicho fin se requiere que durante la fase de diseño y formulación de las propuestas (borrador) de los $\mathrm{POT}^{9}$ (que se desarrolla antes de las etapas de concertación), se integre la EAE, es decir, aplicar el modelo 2 del procedimiento de EAE para así disponer del proyecto del POT con los elementos e insumos ya integrados -derivados de la EAE-, durante las fases de concertación con el público y otras instancias diferentes a las ambientales. La aplicación de la EAE, de acuerdo al modelo 1 de la EIA clásica, no permitiría contar oportunamente con estos resultados. Los 30 días

8 Ver esta fase al inicio del proceso de concertación, en la Gráfica 4

9 En la Gráfica 3 de Fases de Formulación e Implementación de los POTs, ver etapas 1, 2 y 3 especialmente previstos en la legislación (según el procedimiento de la Ley 388 de 1997) para que la AA efectúe la revisión de los POT -desde una perspectiva ambiental- resultarían insuficientes para realizar esta evaluación. Lo anterior supone la aplicación de la EAE desde la fase de formulación del POT. El tipo de EAE que se adapta a este requerimiento es el correspondiente al modelo 2 (acompañamiento de la EAE al proceso de planificación del POT). En la gráfica 5 se hace un esbozo de esta articulación, considerando de forma general -no en detalle- la coordinación entre las fases de la EAE con las etapas de planificación del proyecto del POT.

\section{Conclusión}

Para la adopción de la EAE en Colombia debe reconocerse la falta de unidad y la heterogeneidad

REVISTA CIENTÍFICA / ENERO -DICIEMBRE DE 2010 / No. 12 / BOGOTÁ, D.C. 


\section{GRÁFICA 5. ARTICULACIÓN DEL PROCEDIMIENTO DE LA EAE CON LAS FASES DE FORMULACIÓN /} CONCERTACIÓN DEL POT

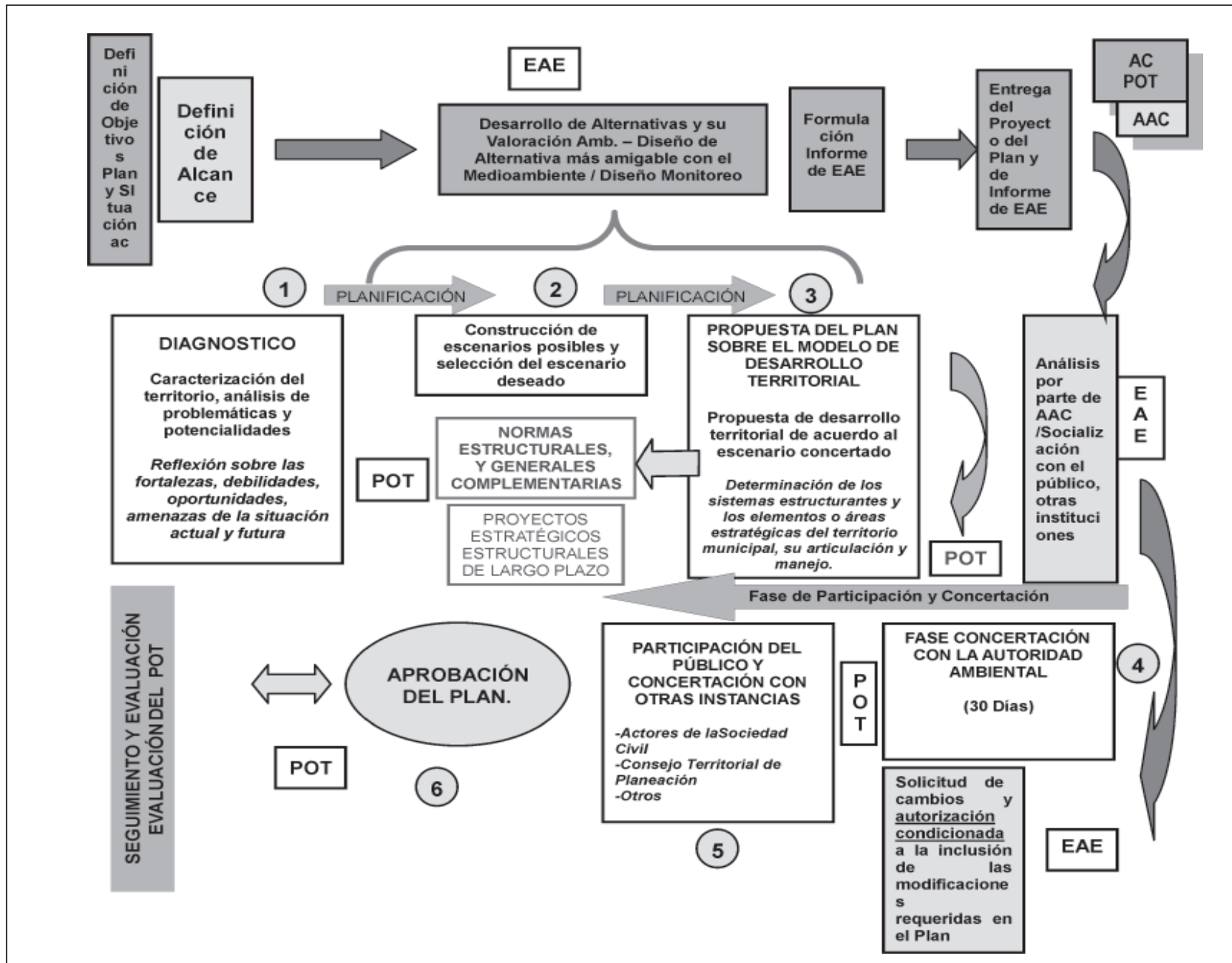

Fuente: elaboración propia basada en la representación de las fases del POT de MinDesarrollo (2001)

característica entre los diferentes campos de las políticas públicas. La ausencia de acuerdos y la carencia de una regulación detallada para gran parte de las área de la participación públicas ha derivado en una variedad instrumental importante en el proceso escalonado de toma de decisiones (en algunos campos existen PPP, en otros sólo algunos de ellos). La falta de acuerdos también se ve reflejada en la diversidad de componentes de las PPP, así como en los diferentes horizontes de tiempo establecidos para estos instrumentos y en los diferentes niveles de concreción, etc. Sólo en algunos campos se presenta formalmente una articulación y un ordenamiento más en detalle en todos los niveles de la jerarquía territorial por parte de estos instrumentos (como es el caso de la planeación del desarrollo). En otros campos se presenta parcialmente la reglamentación formal de algunos de sus instrumentos como, por ejemplo, en el caso del ordenamiento territorial para los POT en la planificación local. Dicha falta de estructuración -precisa- permite un buen margen de discrecionalidad de las autoridades competentes para tomar decisiones unilateralmente. La carencia de una densa reglamentación sobre los procedimientos de formulación, contenidos, plazos, etc. de las PPP debe- 
ría permitir, en principio, una integración más fácil de la EAE (con relación a sus aspectos del procedimiento administrativo) a los sistemas de planificación de PPP existentes ya que no hay en muchos casos una estructura llegalmente formalizada, a la cual debería de adaptarse esta evaluación. Por su parte, es necesario reconocer la dinámica de estas PPP y estructurar, según estos referentes, la articulación de la EAE. Así mismo en los campos del PPP que goza, de una reglamentación detallada, la integración de la $\mathrm{EAE}$, debe reconocer los procedimientos administrativos existentes; éstas aunque no determinan en detalle, si estructura el procedimiento de la EAE a aplicar.

Por otra parte, los principios generales que sirven para estructurar la EIA y la experiencia práctica relacionada con los aspectos del procedimiento administrativo de este instrumento (así como la corta experiencia con la EAE en Colombia), permiten derivar diferentes elementos para estructurar la EAE. Si bien las posibilidades de formular criterios para el procedimiento administrativo de la EAE - a partir de la EIA - no significa que exista una transferencia automática entre ellos. Este análisis permite emplear los aprendizajes obtenidos, a partir del desarrollo, con la evaluación de proyectos para cada uno de los pasos o etapas regulares del procedimiento de la EAE. A partir de la discusión sobre los principios teóricos generales de la evaluación ambiental y de la experiencia con la EIA (y la EAE) en el país, se pueden derivar modelos diferentes para el procedimiento administrativo de la EAE; cada uno (al menos teóricamente) con ventajas y desventajas: uno fundamentado en el procedimiento de la EIA clásica (en este caso el que se aplica en Colombia) y otro - el modelo integrado de EAE- que acompaña los procesos de planificación de PPP. En Colombia, para recomendar la adopción de uno u otro modelo de la EAE, se requeriría efectuar este análisis en diferentes campos de PPP.

Sin embargo, no pareciera tan claro que el modelo del procedimiento actual en Colombia — de EIA clásica con su sucesión de pasos - se pueda implementar como base para estructurar, de forma similar, la serie de etapas del procedimiento de la EAE. Por lo menos, para el ordenamiento territorial, partiendo de los determinantes resultantes de la reglamentación del procedimiento de formulación y aprobación de los POT (nivel local), el procedimiento de la EIA clásica no es adecuado. En consecuencia, es preferible aplicar otro modelo de EAE que permita acompañar el diseño de estos planes, es decir, el modelo de EAE que se articula comparte los diferentes pasos de las fases de planificación y concertación de los POT.

\section{REFERENCIAS BIBLIOGRÁFICAS}

- Handbuch Strategische Umweltprüfung (HB SUP) (Manual de la EAE) Wien: Institut für Techikfolgen - Abshätzung, 1997/2000/2001/2003/2004/2006

- Colombia, Ministerio de Desarrollo (2001), Cartillas sobre Ley 388 de 1997,, Imprenta Nacional.

- Morcillo, P. (2002) La Planeación en ColombiaHistoria, Derecho y Gestión, Bogotá, Universidad Piloto.

- Oñate, J. (2002), La Evaluación Ambiental Estratégica, Madrid, Ediciones Mundiprensa,.

- "Aplicación de Metodología de la Evaluación Ambiental Estratégica al Plan de Exploración y Explotación de Hidrocarburos Costa Afuera en el Mar Caribe" Bogotá: MAVDT - Universidad la Salle, 2006 (Documento trabajado por Jenny Marcela Pineda Pineda -Pasante de la U. de la Salle, bajo la dirección de Marcela Bonilla - MAVDT)

- Constitución Política de Colombia

- Cupei, J. (1994), "Umweltverträglichkeitsprüfung und Entscheidung" en Vorsogen statt nur Schaden Beseitigen, p. 497.

- Decreto Ley 2811 de 1974 (Código de los Recursos Naturales y del Medio Ambiente); Decre-

REVISTA CIENTÍFICA / ENERO -DICIEMBRE DE 2010 / No. 12 / BOGOTÁ, D.C. 
to $1541 / 1978$; Decreto $1594 / 1984$; Decreto 02/1982; Decreto 2104/1983; Decreto-Ley 2655 de 1988 (Código Minero); Decreto 1753 de 1994; Decreto 1768/94, Decreto 1320 de 1998; Decreto 1865/94; Decreto 1128 de 2002; Decreto 1180 de 2003; Decreto 1200 de 2004; Decreto 1220 de 2005; Decreto 2762 de 2005; Decreto 330 de 2007; Decreto 330 de 2007; Decreto 330 de 2007; Decreto 330 de 2007

- Ley 56/1982; Ley 9 de 1989; Ley 99 de 1993; Ley 70 de 1993, Ley 152 de 1994, Ley 388 de 1997; Ley 768 de 2002, Ley 812 de 2003 (Plan Nacional de Desarrollo "Hacia un Estado Comunitario" 2002-2006)

- Documento Mimeo Sistema Nacional de Planificación Bogotá: DNP, 2002

- "EAE de los Planes y Programas del Sector Agropecuario" Bogotá: MAVDT - Sociedad Agricultores de Colombia SAC, 2006

12) Guía Simplificada para la Elaboración del Plan de Ordenamiento Territorial Municipal" Bogotá: IGAC-CAR-KFW-GTZ, 1998, 79p.

- Gómez, J. (1997), Entwicklung der Umweltpolitik in Kolumbien dargestellt am Beispiel der Umwetverträglichkeitsprüfung [tesis doctoral], Berlín, Universidad Técnica de Berlín, Instituto para la Gestión de la Planificación Ambiental.

- Handbuch Strategische Umweltprüfung (HB SUP) (Manual de la EAE) Wien: Institut für Techikfolgen - Abshätzung, 1997/2000/2001/2003/2004/2006

- JACOBY, Christian "Die Strategische Umweltprüfung (SUP) in der Raumplanung” (La EAE en la Planeación Espacial) Berlin: Erich Schmidt Verlag, 2000, 649 p.

- KLÄNE, Christian "Strategische Umweltprüfung (SUP) in der Bauleitplanung" (EAE en la Planeación Directriz para la Construcción - Pla- neación Espacial del Nivel local en Alemania) Hamburg: Verlag Dr. Kovac, 2003, 300 p.

- KLEINSCHMIDT, Volver y WAGNER, Dieter (Comp.) "Strategic Environmental Assessment in Europe - Fourth European Workshop on Environmental Impact Assessment “ Dordrecht (Netherlands): Kluwer Academic Publishers, 1998, 173 p.

- LEE, Norman "Evaluación Ambiental Estratégica aplicada a Políticas, Planes y Programas" en PEINADO, Manuel y SOBRINI, Iñigo "Avances en Evaluación de Impacto ambiental y Ecoauditoría" Madrid: Editorial Trotta, 1997, 567 p.

- Lineamientos para la Formulación e Implementación de un Plan Ambiental Decenal y de una Estrategia Nacional para el Desarrollo Sostenible", Documento, Bogotá: Ministerio de Medio Ambiente, 2001, 25p.

- Londoño, B. (1998), Nuevos instrumentos de participación ambiental, Bogotá D.C., ¿editorial?

- Macías, L. (1998), Introducción al derecho ambiental, Bogotá D.C., Legis Editores.

- "Boletín No 3 - Eje Metodológico" Mesa Permanente de Participación Ciudadana y Comunitaria del Sector Minero Energético Bogotá: Ministerio del Medioambiente, 2002, 23 p.

- Cartillas sobre Ley 388 de 1997 Bogotá: MINDESARROLLO, 2001

- Morcillo, P., (2002), La planeación en Colombiahistoria, derecho y gestión, Bogotá, Universidad Piloto, Facultad de

- NIESTROY, Ingeborg "Die Strategische UVP als Instrument zur Integration von Umweltbelangen in andere Politikbereiche" (La EAE como Instrumento para la Integración de los Intereses Ambientales en otros Campos de Políticas) Berlin: VWF, 2000, 326 P. 
- Nilsson, M. y Jiliberto, R. (2004), "SEA and Decision-Making Sciencies", en Analysing Strategic Environmental Assessment - Towards Better Decision-Making, p. 284.

- Oñate, J. et al. (2002), La evaluación ambiental estratégica, Madrid, Ediciones Mundiprensa.

- Partidario, R. y Clark, R. (2000), Perspectives on Strategic Environmental Assessment, New York, Lewis Publishers.

- Plan Nacional de Desarrollo "Estado Comunitario: Desarrollo para todos" (2006 - 2010) - Texto Conciliado Bogotá: Congreso Nacional, 2007, 118 p.

- "Propuesta conceptual y metodológica para la aplicación de Evaluaciones Ambientales Estratégicas (EAEs) en Colombia" Bogotá: Departamento Nacional de Planeación, 2004, $46 \mathrm{p}$.

- Roth, A. (2003), Políticas públicas; formulación, implementación y evaluación, Bogotá D.C., Ediciones Aurora.

- Schmidt, M.; Joao, E. y Albrech, E. (2005), Implementing Strategic Environmental Assessment, Berlín, Germany: Springer Berlin - Heilderberg.
- "Strategiche Umweltprüfung" Dortmund: UVP Gesellschaft, 1999, 189 p.

- Therivel, R. (2004), Strategic Environmental Assessment in Action, Londres, Earthscan.

- Vargas, A. (1999), El Estado y las políticas públicas, Bogotá D.c., Almudena Editores.

- Vega, L.(2005), Hacia la sostenibilidad ambiental del desarrollo - construcción de pensamiento práctico a través de una política y gestión ambiental sistémica, Bogotá D.C., Coe Ediciones.

- Visión Colombia 2019, II Centenario, Propuesta para Discusión Bogotá: DNP-Ed Planeta, 2005, $384 \mathrm{p}$.

- WEILAND, Ulrike (Hrsg.) "Perspektiven der Raum- und Umweltplanung" (Perspectivas de la Planeación Espacial y Ambiental) Berlin: VWF, 1999, 408 p.

- www.dnp.gov.co/Plan Nacional de Desarrollo 2006 - 2010 (Bases para la discusión), 575 p

- www.mineducacion.gov.co/Balance Plan Decenal de Educación 1996 - 2005 y Plan Decenal Nacional de Educación 2006-2015

- www.upme.gov.co/Presentaciones Día UPME 2007 